\title{
Fabrication and characterization of branched carbon nanostructures
}

\author{
Sharali Malik ${ }^{* 1}$, Yoshihiro Nemoto ${ }^{2}$, Hongxuan $\mathrm{Guo}^{2}$, Katsuhiko Ariga ${ }^{2}$ \\ and Jonathan P. Hill ${ }^{2}$
}

Open Access

\author{
Full Research Paper \\ Address: \\ ${ }^{1}$ Institute of Nanotechnology, Karlsruhe Institute of Technology (KIT), \\ D-76131 Karlsruhe, Germany and ${ }^{2}$ WPI-Center for Materials \\ Nanoarchitectonics, National Institute for Materials Science (NIMS), \\ Namiki 1-1, Tsukuba, Japan \\ Email: \\ Sharali Malik ${ }^{*}$ - sharali.malik@kit.edu \\ * Corresponding author \\ Keywords: \\ branched multiwalled carbon nanotubes; carbon nanostructures; \\ carbon nanotubes; graphene nanoribbons; multiwalled carbon \\ nanotubes
}

\author{
Beilstein J. Nanotechnol. 2016, 7, 1260-1266. \\ doi:10.3762/bjnano.7.116 \\ Received: 04 May 2016 \\ Accepted: 15 August 2016 \\ Published: 05 September 2016 \\ This article is part of the Thematic Series "Advances in nanomaterials II". \\ Guest Editor: H. Hahn \\ (c) 2016 Malik et al.; licensee Beilstein-Institut. \\ License and terms: see end of document.
}

\begin{abstract}
Carbon nanotubes (CNTs) have atomically smooth surfaces and tend not to form covalent bonds with composite matrix materials. Thus, it is the magnitude of the CNT/fiber interfacial strength that limits the amount of nanomechanical interlocking when using conventional CNTs to improve the structural behavior of composite materials through reinforcement. This arises from two wellknown, long standing problems in this research field: (a) inhomogeneous dispersion of the filler, which can lead to aggregation and (b) insufficient reinforcement arising from bonding interactions between the filler and the matrix. These dispersion and reinforcement issues could be addressed by using branched multiwalled carbon nanotubes (b-MWCNTs) as it is known that branched fibers can greatly enhance interfacial bonding and dispersability. Therefore, the use of b-MWCNTs would lead to improved mechanical performance and, in the case of conductive composites, improved electrical performance if the CNT filler was better dispersed and connected. This will provide major benefits to the existing commercial application of CNT-reinforced composites in electrostatic discharge materials (ESD): There would be also potential usage for energy conversion, e.g., in supercapacitors, solar cells and Li-ion batteries. However, the limited availability of b-MWCNTs has, to date, restricted their use in such technological applications. Herein, we report an inexpensive and simple method to fabricate large amounts of branched-MWCNTs, which opens the door to a multitude of possible applications.
\end{abstract}

\section{Introduction}

Lighter, stronger materials such as nanocarbon composites offer benefits for applications such as transport, energy storage/ conversion and bone/tooth replacement. Hence, the mechanical properties of CNTs are utilized in reinforcing polymer composites [1-4], and their electrical conductivity is utilized for conducting polymers [4-6]. Under tensile load only the outermost 
layers of MWCNTs are involved as the relatively weak (van der Waals) bonding between the outer layers and the inner layers leads to slippage - the so called "sword in sheath" failure mechanism, which reduces the load-bearing capacity $[1,7]$. However, under compressive load this slippage leads to the very useful elastic deformation of MWCNTs [8,9].

For all nanoscale reinforcing component materials (NRCMs) including nanocarbons such as graphene, there remain two wellknown, long standing issues which are widely recognized as being critical for the development of mechanically efficient nanocomposites: a) inhomogeneous dispersion of the filler [10] and (b) insufficient strength of the interphase between the filler and the matrix [11]. However, these issues can be addressed by utilizing branched multiwalled carbon nanotubes (b-MWCNTs). It is known from theory and simulation experiments [12-14] that branched fibers can greatly enhance interfacial bonding and dispersability. Such an approach is exemplified by the process of adding straw (branched plant fibers) to mud to make stronger bricks which has been used since the Neolithic period, i.e., before $3400 \mathrm{BC}$ [15]. More recently, Masselter et al. have correlated the functional morphology of branching in plants with mechanical behavior and concluded that the concepts generated have a high potential for implementation in the development of branched fiber-reinforced technical composites [16] With respect to electrical and electronic properties, it is well known that in carbon nanotube networks the junction resistance controls the overall performance [17]. Therefore, in addition to b-MWCNTs/composite applications, the enhanced electrical properties of networks arising within this new material has major potential benefits for design, development and production of supercapacitors, solar cells and Li-ion batteries.

The first experimental observation of branched carbon nanotubes appears to have been in 1995, when after using an arc-discharge method L-, Y- and T-shaped MWCNTs were produced [18]. Subsequently, branched CNTs have been fabricated using a variety of methods, which include pyrolysis of metallocenes $[19,20]$, nanowelding [21], catalytic CVD [22,23], carbon infiltration of MWCNTs [24], templating [25] and chemical functionalization [26]. However, none of these methods are easily industrially scalable. Herein, we report a cheap and simple method to fabricate large amounts of branched MWCNTs in order to address the well-known problems of adequate dispersion and sufficiently strong interfacial bonding required for large-scale applications.

As a starting material it was decided to use the commercially available MWCNTs, namely Baytubes ${ }^{\circledR}$. Other workers have shown using high resolution transmission electron microscopy (HRTEM) that Baytubes ${ }^{\complement}$ are parallel-walled in long closed sections and that they are strong and relatively pure [27]. They form around a catalyst and are supplied as loosely agglomerated pellets (Figure 1), which has advantages regarding health, safety and environment. According to the data sheet Baytubes ${ }^{\circledR}$ belong to the category of short, thin tangled MWCNTs for which no "fibre-like" pathogenic behaviour is expected based on the available data. The MWCNTs used in this research were Baytubes ${ }^{\odot}$ C 150P (Bayer Material Science A.G., Leverkusen, Germany).

\section{Results and Discussion}

The idea of this work is to "unzip" the MWCNTs and to allow them to re-roll and form branched MWCNTs. This works for MWCNTs like Baytubes ${ }^{\complement}$, because they have parallel sides [27] and so they can unzip to form layered nanoribbons. Since they are in long closed sections (Figure 1d) we can assume that the end of these sections acts like a "rip-stop" so that the unzipped tubes can re-roll from these points to form branched structures (Figure 2a-c). The Raman spectra of the as-received MWCNTs (bottom spectrum) and of the branched-MWCNTs (top spectrum) are shown in (Figure 2d). Both sets of spectra are typical of MWCNTs and the $I_{\mathrm{D}} / I_{\mathrm{G}}$ values $[28,29]$ of the as-received MWCNTs and the b-MWCNTs are 1.26 and 1.43, respectively. This indicates that they both have very similar defect densities.

The mechanism of unzipping MWCNTs to form graphene nanoribbons is well known from research by Hirsch [30] and Dai et al. [31] but the procedure is complex and the yield is low [32-34]. However, as we show here, if the aim is to make branched-MWCNT then the procedure is much simpler. Thus, as-received MWCNTs were heated to $500{ }^{\circ} \mathrm{C}$, which results in the introduction of defects that later act as the "unzipping" points. The procedure has the additional benefit of cleaning the tubes as substantiated from the Raman (Figure 2d) and HRTEM data (Figure 3) confirming the absence of carbon impurities or residual catalyst material. The heated MWCNTs were then sonicated in ethanol which causes the MWCNTs to unzip and re-roll. Figure 4 shows a suggested schematic sequence in agreement with observations by Kaner et al. [35] and Geim [36] indicating that nanoribbons tend to re-roll unless prevented from doing so.

Dispersions of the starting MWCNTs and b-MWCNTs were compared by dispersing $1 \mathrm{mg}$ of each material in $4 \mathrm{~mL}$ of ethanol. These dispersions were then centrifuged at $3500 \mathrm{~g}$ for $2 \mathrm{~h}$ and then left to stand for $24 \mathrm{~h}$. The liquid suspensions are shown in Figure 5. The b-MWCNTs clearly show better dispersability compared to the starting MWCNTs.

As no surfactants or expensive polymers are needed for this process, it can be described as inexpensive and easy and it 

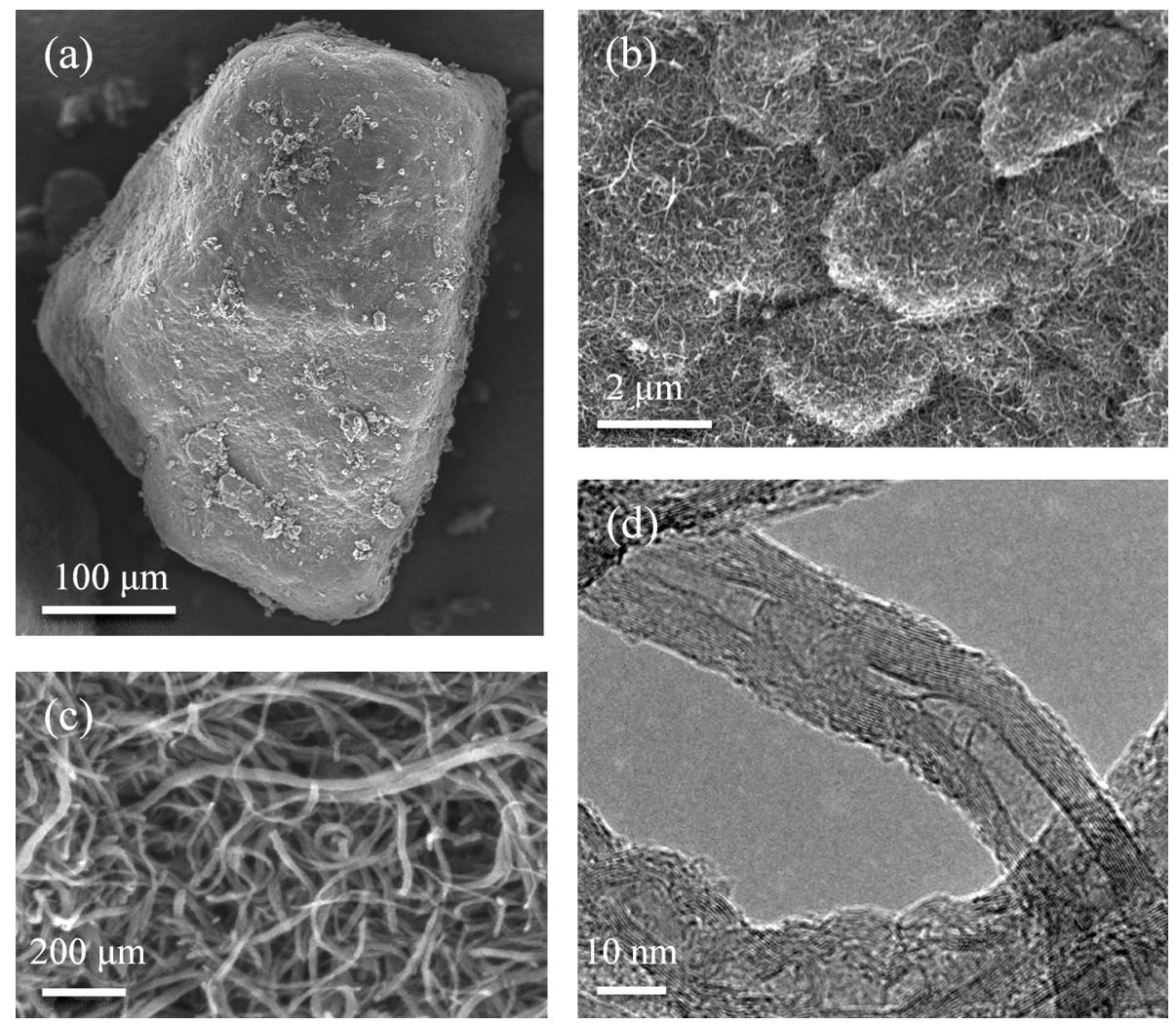

Figure 1: a) SEM overview of a Baytubes agglomerated pellet; b, c) SEM details of the MWCNTs; d) TEM detail of typical MWCNTs.
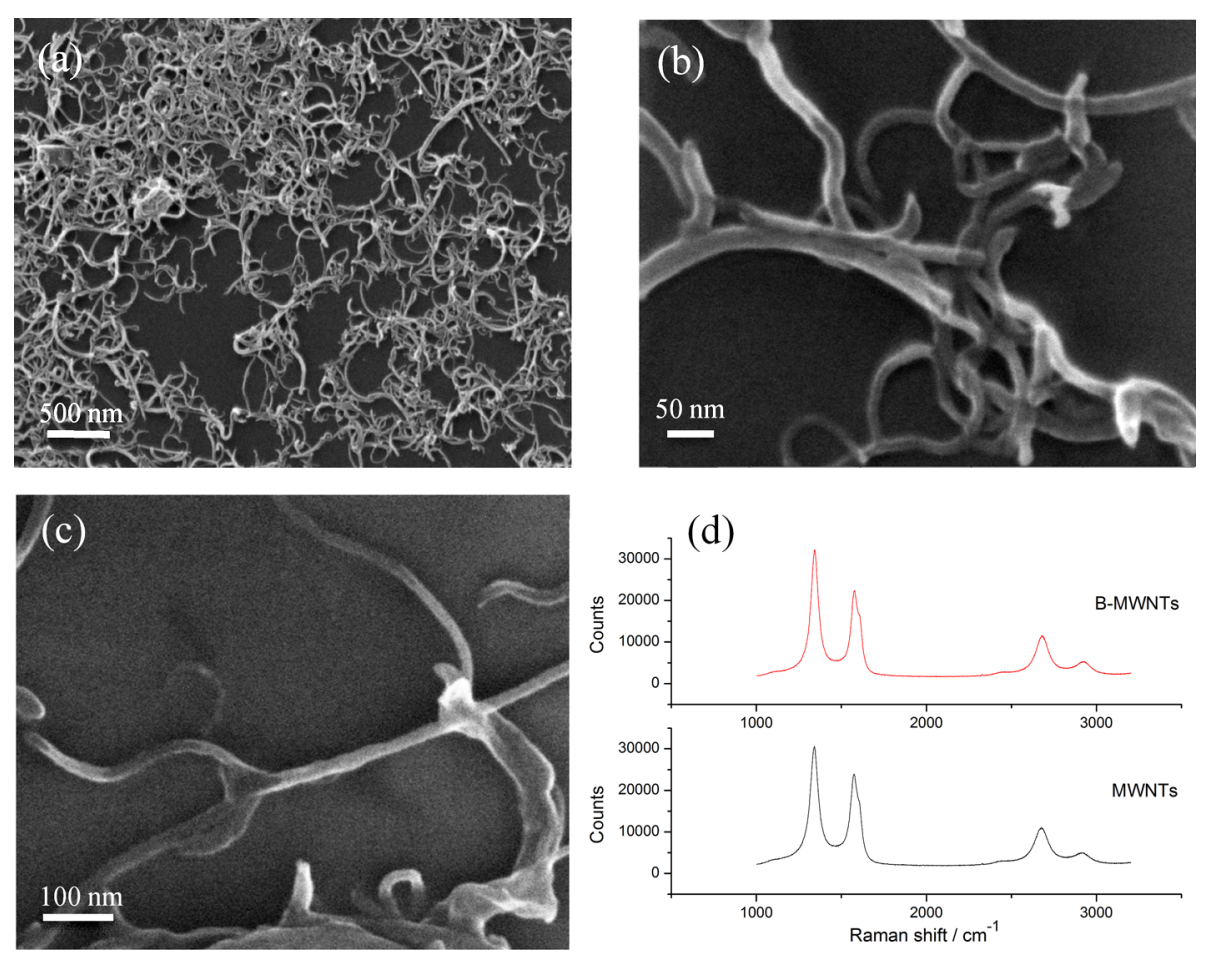

Figure 2: a) Helium ion microscope (HeIM) overview of b-MWCNTs and b) HelM detail of b-MWCNTs; c) SEM detail of unzipped and branchedMWCNTs; d) Raman spectra of as received MWCNTs (bottom spectrum) and b-MWCNTs (top spectrum) - both at $532 \mathrm{~nm}$. 

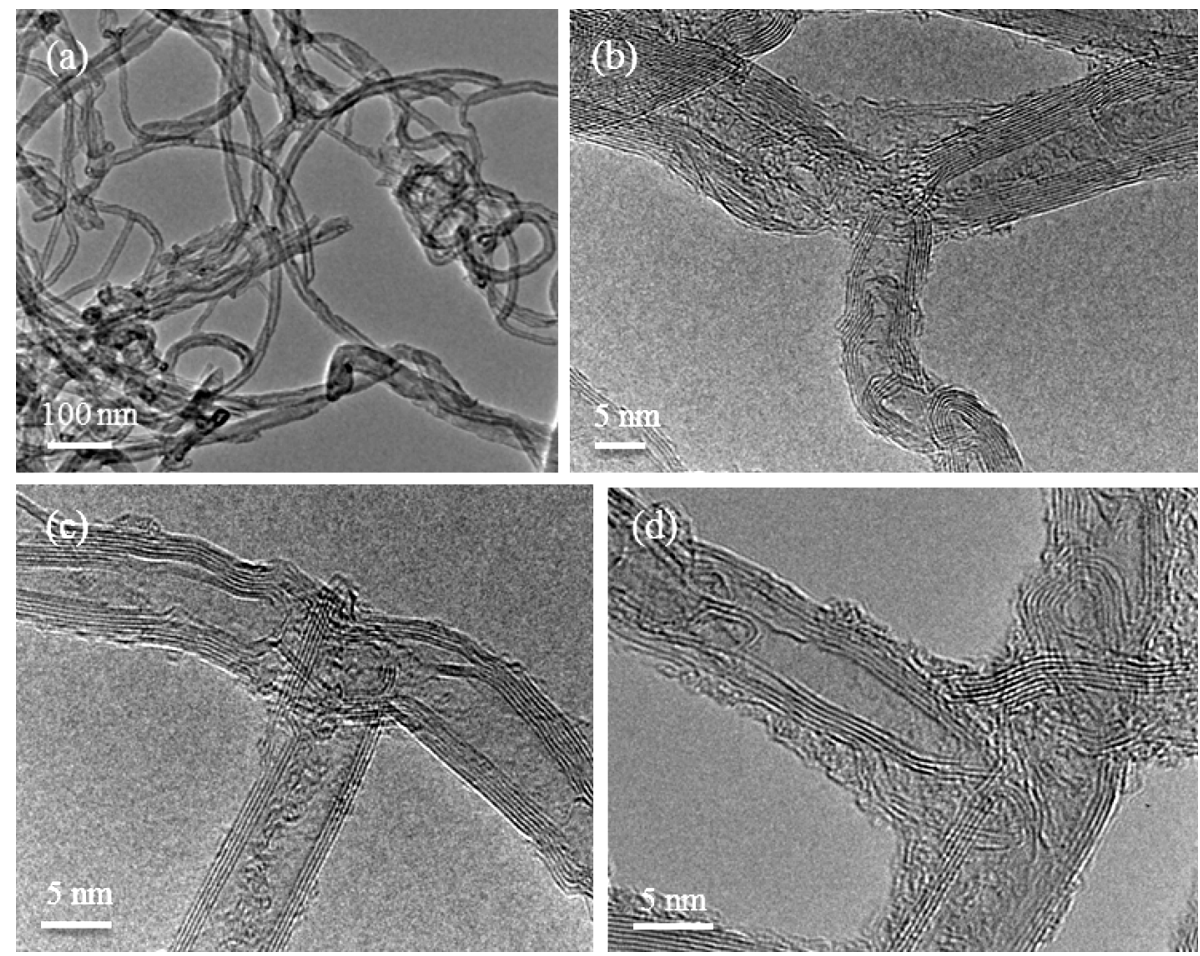

Figure 3: a) HRTEM overview of branched-MWCNTs and b) HRTEM detail of Y-pattern b-MWCNTs; c, d) HRTEM detail of T-pattern b-MWCNTs.

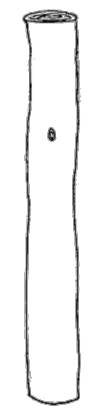

a

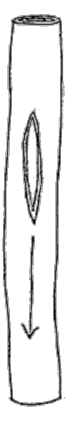

b

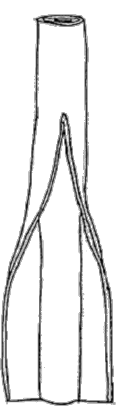

c

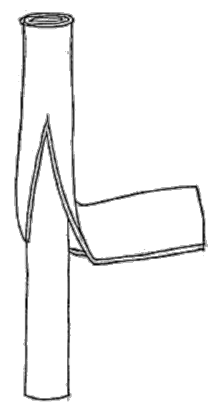

d

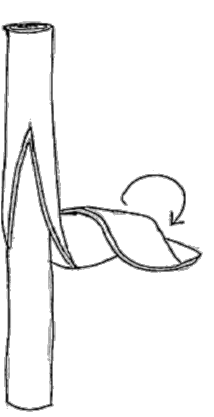

e
Figure 4: A schematic diagram of the suggested "unzipping" and "rerolling" sequence: a) formation of unzipping point; b) onset of unzipping; c) unzipping and onset of peeling of inner parallel tubes; d) outer layers peeling out as a sheet; e) onset of re-rolling of outer layers.

results in clean b-MWCNTs (Figure 3). The yield is estimated (using the methodology by Dai et al. [31]) to be about $60 \%$ branched MWCNTs. The Y-branched MWCNTs fabricated here (Figure 3b) obey geometric conservation laws that are consistent with earlier theory [37] in that the angle between two neighboring branches should be $120^{\circ}$. Therefore, in this instance theory and experiment are in agreement, even though this agreement is not "perfect" as in practice defects will certainly be present.

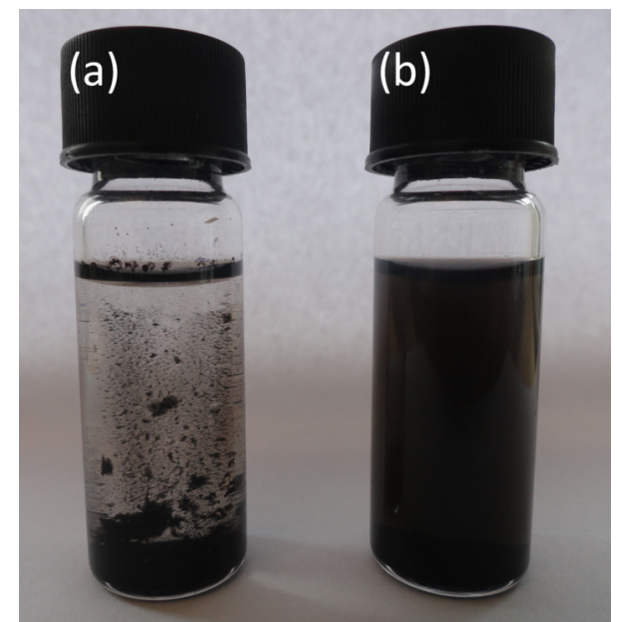

Figure 5: (a) Dispersion of MWCNTs starting material; (b) dispersion of b-MWCNTs.

The unzipping and re-rolling process appears to reduce the number of walls on the MWCNTs compared to those for the as-received tubes (Figure 1d) and it is also possible that some of the tubes re-roll to form carbon nano-scrolls (CNS) [35,38-40]. This possibility is evidenced in Figure $3 \mathrm{c}$ where the number of walls of the three parts of the " $T$ " pattern does not meet the "Russian Doll" MWCNTs requirement of $N($ left $)=N($ right $)+$ 
$N($ down $)$ or $N($ right $)=N($ left $)+N($ down $)$. The Raman spectra show that the b-MWCNTs have a similar defect density as the starting material, which shows that the material has not been damaged by the fabrication procedure. The EDX analysis of the MWCNTs starting material is $100 \%$ carbon (the data sheet indicates a carbon purity of over 95\%). The EDX analysis of the branched-MWNTs is $96 \%$ carbon and $4 \%$ oxygen. This is consistent with the initial heating in air, which removes amorphous impurities and etches/oxidizes the MWCNTs at defect sites without damaging the sidewalls [31]. In many nanocarbon material applications the presence of residual surfactant or organic residues can be a problem, but this is not the case here.

The experimental procedure produces b-MWCNTs when using thick MWCNTs (more than a few walls). However, when the same procedure is used with thin MWCNTs (e.g., triple-walled MWCNTs, Figure 6a), synthesised by a "water-assisted" CVD method [41], graphene nanoribbons are produced in small yields (Figure 6b) which is consistent with earlier works by Hirsch [30] and Dai et al. [31].

\section{Conclusion}

In summary, we have described herein a new, simple and cheap route to fabricate large amounts of branched MWCNTs using widely available commercial MWCNTs. We have demonstrated a facile procedure for making clean branched nanostructures and this opens promising avenues for the development and manufacturing of nanocarbon composites for a variety of commercial applications. The fabrication and testing of composite materials with branched MWCNTs as well as measurements of electrical conductivity are currently in progress.

\section{Experimental}

The MWCNTs used in this research were Baytubes ${ }^{\complement} \mathrm{C}$ 150P (Lot-No E0009AA007, Drum-No 033), supplied by Bayer Material Science A.G. (Leverkusen, Germany).

\section{Preparation of b-MWCNTs}

Commercial MWCNTs were heated in air to $500{ }^{\circ} \mathrm{C}$ for $2 \mathrm{~h}$ to give the "stage I"-modified MWCNTs. Then a small amount of these were sonicated in ethanol $(30 \mathrm{~mL}$ ) for $8 \mathrm{~h}$ (ultrasonic denerator, model GSCVP 150 at ca. $80 \%$ power). The dispersion was centrifuged at $3500 \mathrm{~g}$ for $90 \mathrm{~min}$ to give the "stage II"modified MWCNTs, which are the b-MWCNTs. The supernatant was spotted onto lacey carbon $\mathrm{Cu}$ TEM grids and onto polished Si chips for subsequent characterization.

\section{Preparation of graphene nanoribbons}

A "water-assisted" CVD process as reported earlier [41,42] was used to fabricate thin walled MWCNTs (ca. three walls), which were subsequently heated in air to $500{ }^{\circ} \mathrm{C}$ for $2 \mathrm{~h}$. Subsequently, ca. $10 \mathrm{mg}$ of this material was sonicated (UP200s Dr. Hielscher, $200 \mathrm{~W}, 24 \mathrm{~Hz}, 0.5$ cycles, $60 \%$ amplitude) in ethanol ( $4 \mathrm{~mL}$ ) for $1 \mathrm{~h}$. The resultant dispersion was spotted onto plain $\mathrm{Cu}$ TEM grids and polished Si chips for subsequent characterization.

\section{Characterization}

Quality and morphology of the fabricated branched nanostructures were scrutinised by Raman spectroscopy (Renishaw inVia), TEM (Tecnai F20 ST at $200 \mathrm{kV}$ ), HRTEM (Jeol ARM at $120 \mathrm{kV}$ ), SEM (Zeiss Ultra-Plus at $5 \mathrm{kV}$ ), SEM (Zeiss Leo 1530 at $10 \mathrm{kV}$ with Oxford X-Max $50 \mathrm{EDX}$ ) and helium ion microscopy (HeIM, Zeiss Orion at $30 \mathrm{kV}$ ).

\section{Acknowledgements}

This paper is dedicated to Professor Herbert Gleiter on the occasion of his 75 th birthday. S. M. acknowledges the continuing support of Prof. Dr. M. M. Kappes. This work was partly supported by World Premier International Research Center Initiative (WPI Initiative) from MEXT, Japan and we thank Dr. Daisuke Fuijita and Dr. Kiyotaka Iiayma for their support. We acknowledge Dr. Tony D. Keene, Southampton University, for his support. We also thank Bayer Material Science A.G. for
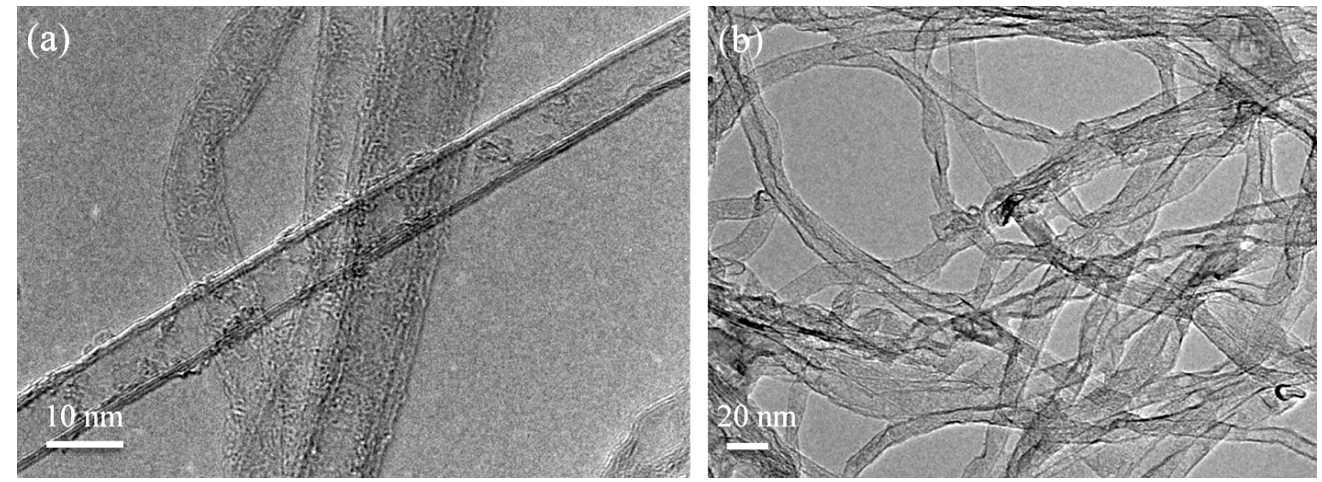

Figure 6: TEM overview of a) thin MWCNTs starting material and b) graphene nanoribbons after treatment. 
supply of MWCNTs. The authors would like to acknowledge networking support by the COST Action CA15107 (MultiComp).

\section{References}

1. Yu, M.-F.; Lourie, O.; Dyer, M. J.; Moloni, K.; Kelly, T. F.; Ruoff, R. S. Science 2000, 287, 637-640. doi:10.1126/science.287.5453.637

2. Peng, B.; Locascio, M.; Zapol, P.; Li, S.; Mielke, S. L.; Schatz, G. C.; Espinosa, H. Nat. Nanotechnol. 2008, 3, 626-631. doi:10.1038/nnano.2008.211

3. Wong, E. W.; Sheehan, P. E.; Lieber, C. M. Science 1997, 277, 1971-1975. doi:10.1126/science.277.5334.1971

4. Hernándey-Pérez, A.; Avilés, F.; May-Pat, A.; Valadez-González, A.; Herrera-Franco, P. J.; Bartolo-Pérez, P. Compos. Sci. Technol. 2008, 68, 1422-1431. doi:10.1016/j.compscitech.2007.11.001

5. Gojny, F. H.; Wichmann, M. H. G.; Fiedler, B.; Kinloch, I. A.; Bauhofer, W.; Windle, A. H.; Schulte, K. Polymer 2006, 47, 2036-2045. doi:10.1016/j.polymer.2006.01.029

6. Bauhofer, W.; Kovacs, J. Z. Compos. Sci. Technol. 2009, 69, 1486-1498. doi:10.1016/j.compscitech.2008.06.018

7. Ganesan, Y.; Peng, C.; Lu, Y.; Loya, P. E.; Moloney, P.; Barrera, E.; Yakobson, B. I.; Tour, J. M.; Ballarini, R.; Lou, J.

ACS Appl. Mater. Interfaces 2011, 3, 129-134. doi:10.1021/am1011047

8. Schadler, L. S.; Giannaris, S. C.; Ajayan, P. M. Appl. Phys. Lett. 1998, 73, 3842-3844. doi:10.1063/1.122911

9. Wagner, H. D.; Ajayan, P. M.; Schulte, K. Compos. Sci. Technol. 2013, 83, 27-31. doi:10.1016/j.compscitech.2013.04.017

10. Qian, D.; Dickey, E. C.; Andrews, R.; Rantell, T. Appl. Phys. Lett. 2000, 76, 2868-2870. doi:10.1063/1.126500

11. Wagner, H. D.; Lourie, O.; Feldman, Y.; Tenne, R. Appl. Phys. Lett. 1998, 72, 188-190. doi:10.1063/1.120680

12. Fu, S.; Zhou, B.; Lung, C. Compos. Sci. Technol. 1993, 47, 245-250. doi:10.1016/0266-3538(93)90033-D

13. Suzuki, T.; Miyajima, T.; Sakai, M. Compos. Sci. Technol. 1994, 51, 283-289. doi:10.1016/0266-3538(94)90197-X

14. Liu, L.; Zhang, L.; Lua, J. Appl. Phys. Lett. 2012, 101, 161907. doi:10.1063/1.4761936

15. Brownell, W. E. Applied Minerology; Structural Clay Products, Vol. 9; Springer: Vienna, 1976; pp 1-23. doi:10.1007/978-3-7091-8449-3_1

16. Masselter, T.; Eckert, S.; Speck, T. Beilstein J. Nanotechnol. 2011, 2 , 173-185. doi:10.3762/bjnano.2.21

17. Nirmairaj, P. N.; Lyons, P. E.; De, S.; Coleman, J. N.; Boland, J. J. Nano Lett. 2009, 9, 3890-3895. doi:10.1021/n19020914

18. Zhou, D.; Seraphin, D. S. Chem. Phys. Lett. 1995, 238, 286-289. doi:10.1016/0009-2614(95)00406-T

19. Deepak, F. L.; John, N. S.; Govindaraj, A.; Kulkarni, G. U.; Rao, C. N. R. Chem. Phys. Lett. 2005, 411, 468-473. doi:10.1016/j.cplett.2005.06.076

20. Wei, Q.; Liu, Y.; Zhang, L.; Huang, S. Nano-Micro Lett. 2013, 5, 124-128. doi:10.1007/BF03353739

21. Rodríguez-Manzo, J. A.; Wang, M.-S.; Banhart, F.; Bando, Y.; Golberg, D. Adv. Mater. 2009, 21, 4477-4482. doi:10.1002/adma.200901321

22. Heyning, O. T.; Bernier, P.; Glerup, M. Chem. Phys. Lett. 2005, 409, 43-47. doi:10.1016/j.cplett.2005.04.097
23. Romo-Herrera, J. M.; Sumpter, B. G.; Cullen, D. A.; Terrones, H.; Cruz-Silva, E.; Smith, D. J.; Meunier, V.; Terrones, M. Angew. Chem., Int. Ed. 2008, 47, 2948-2953. doi:10.1002/anie.200705053

24. Jin, Y.; Zhang, Y.; Zhang, Q.; Zhang, R.; Li, P.; Qian, W.; Wei, F. Nanoscale 2013, 5, 6181-6186. doi:10.1039/c3nr01069d

25. Meng, G.; Han, F.; Zhao, X.; Chen, B.; Yang, D.; Liu, J.; Xu, Q.; Kong, M.; Zhu, X.; Jung, Y. J.; Yang, Y.; Chu, Z.; Ye, M.; Kar, S.; Vajtai, R.; Ajayan, P. M. Angew. Chem., Int. Ed. 2009, 48, 7166-7170. doi:10.1002/anie.200901999

26. Balaban, T. S.; Balaban, M. C.; Malik, S.; Hennrich, F.; Fischer, R.; Rösner, H.; Kappes, M. M. Adv. Mater. 2006, 18, 2763-2767. doi:10.1002/adma.200600138

27. Tessonnier, J.-P.; Rosenthal, D.; Hansen, T. W.; Hess, C.; Schuster, M. E.; Blume, R.; Girgsdies, F.; Pfänder, N.; Timpe, O.; Su, D. S.; Schlögl, R. Carbon 2009, 47, 1779-1798. doi:10.1016/j.carbon.2009.02.032

28. Ouyang, Y.; Cong, L. M.; Chen, L.; Liu, Q. X.; Fang, F. Physica E 2008, 40, 2386-2389. doi:10.1016/j.physe.2007.11.008

29. Dresselhaus, M. S.; Jorio, A.; Souza Filho, A. G.; Saito, R. Philos. Trans. R. Soc. London, Ser. A 2010, 368, 5355-5377. doi:10.1098/rsta.2010.0213

30. Hirsch, A. Angew. Chem., Int. Ed. 2009, 48, 6594-6596. doi:10.1002/anie.200902534

31. Jiao, L.; Wang, X.; Diankov, G.; Wang, H.; Dai, H. Nat. Nanotechnol. 2010, 5, 321-325. doi:10.1038/nnano.2010.54

32. Li, X.; Wang, X.; Zhang, L.; Lee, S.; Dai, H. Science 2008, 319, 1229-1232. doi:10.1126/science.1150878

33. Kosynkin, D. V.; Higginbotham, A. L.; Sinitskii, A.; Lomeda, J. R.; Dimiev, A.; Price, B. K.; Tour, J. M. Nature 2009, 458, 872-876. doi:10.1038/nature07872

34. Jiao, L.; Zhang, L.; Wang, X.; Diankov, G.; Dai, H. Nature 2009, 458, 877-880. doi:10.1038/nature07919

35. Viculis, L. M.; Mack, J. J.; Kaner, R. B. Science 2003, 299, 1361. doi:10.1126/science. 1078842

36. Geim, A. K. Science 2009, 324, 1530-1534. doi:10.1126/science.1158877

37. Yin, Y.; Chen, Y.; Yin, J.; Huang, K. Nanotechnology 2006, 17, 4941-4945. doi:10.1088/0957-4484/17/19/027

38. Braga, S. F.; Coluci, V. R.; Legoas, S. B.; Giro, R.; Galvão, D. S.; Baughman, R. H. Nano Lett. 2004, 4, 881-884. doi:10.1021/nl0497272

39. Mpourmpakis, G.; Tylianakis, E.; Froudakis, G. E. Nano Lett. 2007, 7, 1893-1897. doi:10.1021/nl070530u

40. Shi, X.; Pugno, N. M.; Gao, H. Acta Mech. Solida Sin. 2010, 23 , 484-497. doi:10.1016/S0894-9166(11)60002-5

41. Kiowsky, O.; Lebedkin, S.; Hennrich, F.; Malik, S.; Rösner, H.; Arnold, K.; Sürges, C.; Kappes, M. M. Phys. Rev. B 2007, 75, 075421. doi:10.1103/PhysRevB.75.075421

42. Hata, K.; Futuba, D. N.; Mizuno, K.; Namai, T.; Yumura, M.; lijima, S. Science 2004, 306, 1362-1364. doi:10.1126/science.1104962 


\section{License and Terms}

This is an Open Access article under the terms of the Creative Commons Attribution License

(http://creativecommons.org/licenses/by/4.0), which permits unrestricted use, distribution, and reproduction in any medium, provided the original work is properly cited.

The license is subject to the Beilstein Journal of Nanotechnology terms and conditions:

(http://www.beilstein-journals.org/bjnano)

The definitive version of this article is the electronic one which can be found at:

doi:10.3762/bjnano.7.116 